\title{
Changing inpatient diabetes care in a district general hospital
}

\author{
MARIE WALLNER, ${ }^{1}$ BASHARAT ANDRABI, ${ }^{1}$ DAVID RUSSELL-JONES, ${ }^{2}$ ROSELLE HERRING ${ }^{2}$
}

\begin{abstract}
Introduction: People with diabetes in hospital have longer lengths of stay and are at higher risk of experiencing avoidable harm. This has a significant impact on patient flow and capacity in any hospital Trust.

Aims and Methods: A Trust-wide peripatetic inpatient diabetes service redesign was performed to deliver reduced medication errors, improved patient flow, reduced length of stay and reduced inpatient risk. The service redesign was delivered without new recurring expenditure on senior staff. The model of care was multidisciplinary and introduced consensus and evidence-based care with clear governance processes.

Results: Following introduction of the new service on 7 December 2017 to 1 June 2018, a reduction in length of stay in both medicine and surgical divisions was seen with 2,168 'saved' inpatient bed days compared with the same time period in the preceding year, which represented a significant cost saving for the Trust and improvement in patient flow. This was associated with a reduction in the number of diabetes-related Datix reports and serious untoward incidents. Conclusions: This is the first major diabetes service redesign in a small district general hospital. The introduction of a dedicated inpatient diabetes service has led to Trust-wide improvements in patient care and patient flow without additional cost to the Trust.
\end{abstract}

Br J Diabetes 2019;19:49-52

Key words: diabetes, inpatient, length of stay, strategy, district general hospital, patient harm

\section{Background}

The NHS in England spends around $£ 2.5$ billion a year on inpatient care for people with diabetes (approximately $11 \%$ of total NHS inpatient expenditure). ${ }^{1}$ People with diabetes tend to have longer hospital admissions and are more often admitted overnight for

Royal Surrey County Hospital, Guildford, Surrey, UK

Diabetes Specialist Registrar, Diabetes and Endocrinology Department

2 Consultant Diabetologist

Address for correspondence: Dr Marie Wallner Royal Surrey County Hospital, Egerton Road, Guildford, Surrey GU2 7XX, UK

E-mail: marie.wallner1@nhs.net

https://doi.org/10.15277/bjd.2019.212 planned procedures. In spite of these high levels of expenditure, there is evidence of variation in care. People with diabetes experience avoidable harm while in hospital due to increased hypoglycaemic episodes and inpatient diabetic ketoacidosis. ${ }^{2}$ Reasons for this are complex and can include inappropriate insulin treatment or lack of carbohydrate. ${ }^{3}$

Royal Surrey County Hospital (RSCH) is a district general hospital. The 2017 National Diabetes Inpatient Audit (NaDIA) identified that $\mathrm{RSCH}$ had an inpatient diabetes prevalence of 16\% (66 patients with diabetes in 412 audited beds). ${ }^{2}$ The average length of stay (LOS) from 1 December 2016 to 30 November 2017 showed the Medicine directorate LOS for people with diabetes was 8.52 days compared with 6.77 days for those without diabetes. In the Surgery and Oncology directorate the LOS for people with diabetes was 5.27 days compared with 4.34 days for people without diabetes. This had a significant impact on patient flow and capacity within the Trust. Furthermore, diabetes medication errors were significantly higher than the national average and $\mathrm{RSCH}$ was in the highest quartile for incidence of all inpatient hypoglycaemia and severe hypoglycaemia. ${ }^{2}$ Diabetes UK also reported that people with diabetes admitted to RSCH had an excess mortality between 1 April 2010 and 31 March 2012.4 Although the overall mortality for inpatients with diabetes was slightly better than the national average, the mortality rate for patients without diabetes was extremely low; consequently, the excess mortality associated with diabetes was one of the highest in the UK. ${ }^{4}$

A Trust-wide peripatetic inpatient diabetes service redesign was agreed that would refocus the resources of the Diabetes and Endocrinology directorate towards the care of inpatients with diabetes and away from a 16-bed general medical ward. This approach was ambitious. It had been used elsewhere in large UK centres but not in a district general hospital. ${ }^{5}$ The initiative aimed to enable the Trust to deliver reduced medication errors, improved patient flow, reduced length of stay and reduced inpatient risk. Importantly, the service aligned to the vision of the Diabetes UK multidisciplinary team ${ }^{6}$ and new recurring expenditure on senior staff was not required.

\section{Methods}

The study design was interventional: 6 months of pre-intervention observational data were compared with 6 months postintervention outcomes.

In 2017 the NaDIA showed that only $20.3 \%$ of people admitted with diabetes were seen by a member of the diabetes team. ${ }^{2}$ Inpatients were seen by a diabetes specialist nurse following a ward referral and by a diabetes consultant or specialist registrar if there were difficult management issues. No remote 
blood glucose monitoring was undertaken and there was no formal way to identify people admitted to hospital with diabetes or proactively to audit diabetes harm.

The intervention involved restructuring resources to support a consultant-led multidisciplinary inpatient team. Members included diabetes consultants, diabetes specialist nurses, diabetes specialist registrar, support from a medical coder and data analyst, access to specialist podiatrists, ward pharmacists and ward dieticians. The diabetes consultants transferred their inpatient programmed activities allocation from the general medical ward to support the new inpatient diabetes service. Consultant outpatient activity remained the same and the 1.6 Whole Time Equivalent diabetes specialist nurses restructured their support to focus on a greater inpatient education role for healthcare professionals and patients.

A new Acute Frailty Unit was set up at no additional cost in the 16-bed general medical ward staffed by the junior doctors, nurses and allied healthcare professionals previously allocated to the Diabetes and Endocrinology general medical ward, which was in keeping with another key Trust strategy. The Frailty consultants transferred their programmed activities allocation from a peripatetic service to the new Acute Frailty Unit.

\section{The inpatient model}

Every person with diabetes was identified and reviewed remotely through the Freestyle Precision Pro Blood Glucose and beta Ketone Monitoring System (Abbott Diabetes Care Ltd, Witney, Oxon, UK). A daily ward round list was then generated for people who needed a direct review. This included everyone with type 1 diabetes, pancreatic diabetes, people who have had pancreatic surgery or pancreatitis, those with a primary diabetes foot problem, people with type 2 diabetes with evidence of hyperglycaemia (blood glucose $>16 \mathrm{mmol} / \mathrm{L}$ ) or hypoglycaemia (blood glucose $<4 \mathrm{mmol} / \mathrm{L}$ ), people with ongoing inpatient management issues and those who had been referred directly by ward teams or a direct patient referral.

\section{Introduction of governance process}

Any inpatient death of a person with diabetes was reviewed in a monthly morbidity and mortality meeting. Complex discharges were coordinated via a weekly diabetes multidisciplinary team with the community diabetes nurses. Medical coders and data analysts developed a diabetes outcome dashboard with discharge data and inpatient harms in accordance with NaDIA-Harms data collection. ${ }^{7}$

\section{Results}

The multidisciplinary inpatient diabetes team started on 7 December 2017. Every person admitted to hospital with diabetes was reviewed remotely on a daily basis. On average, 30 patients a day were identified as benefiting from direct patient review by a member of the multidisciplinary inpatient team.

The inpatient glucose data are shown in Table 1. There was little variation in the number of people who experienced hyperglycaemia (blood glucose $>16 \mathrm{mmol}$ ) or hypoglycaemia (blood glucose $<4 \mathrm{mmol}$ ) with the introduction of the service. However, there was an improvement in occupied bed days in both Medicine and Surgical directorates. Matched data were compared for the 6 months prior to the intervention and 6 months post intervention. In the Medicine directorate there was a total of 10,271

Table 1 Daily capillary blood glucose levels for inpatients with a primary or secondary diagnosis of diabetes

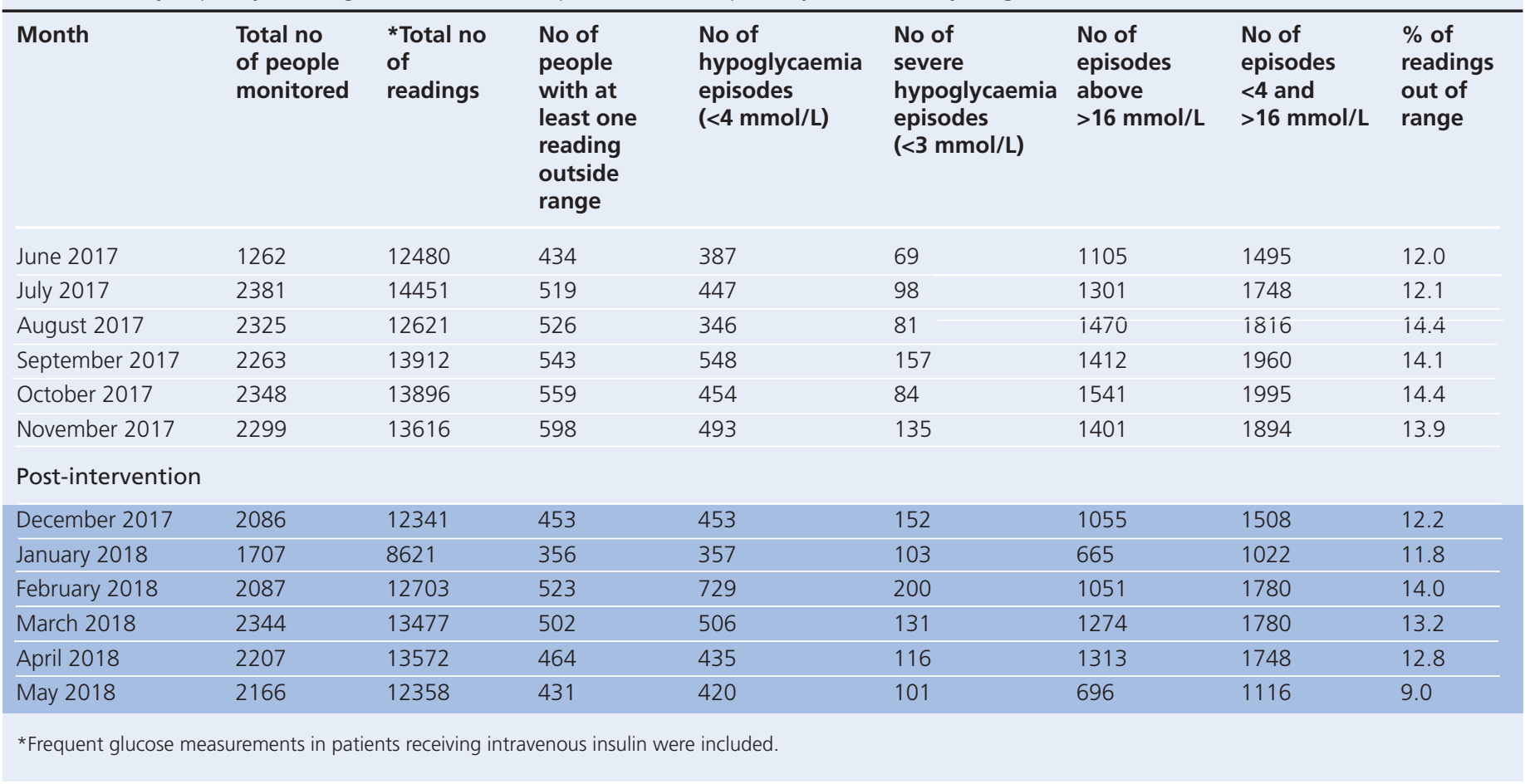


Figure 1. Occupied bed days for people with diabetes following introduction of multidisciplinary inpatient team. (A) Occupied bed days in Access and Medicine for people with diabetes following introduction of multidisciplinary inpatient team from 7 December 2017 to 1 June 2018. (B) Occupied bed days in Surgery (elective and emergency) for people with diabetes following introduction of multidisciplinary inpatient team in 2017-2018

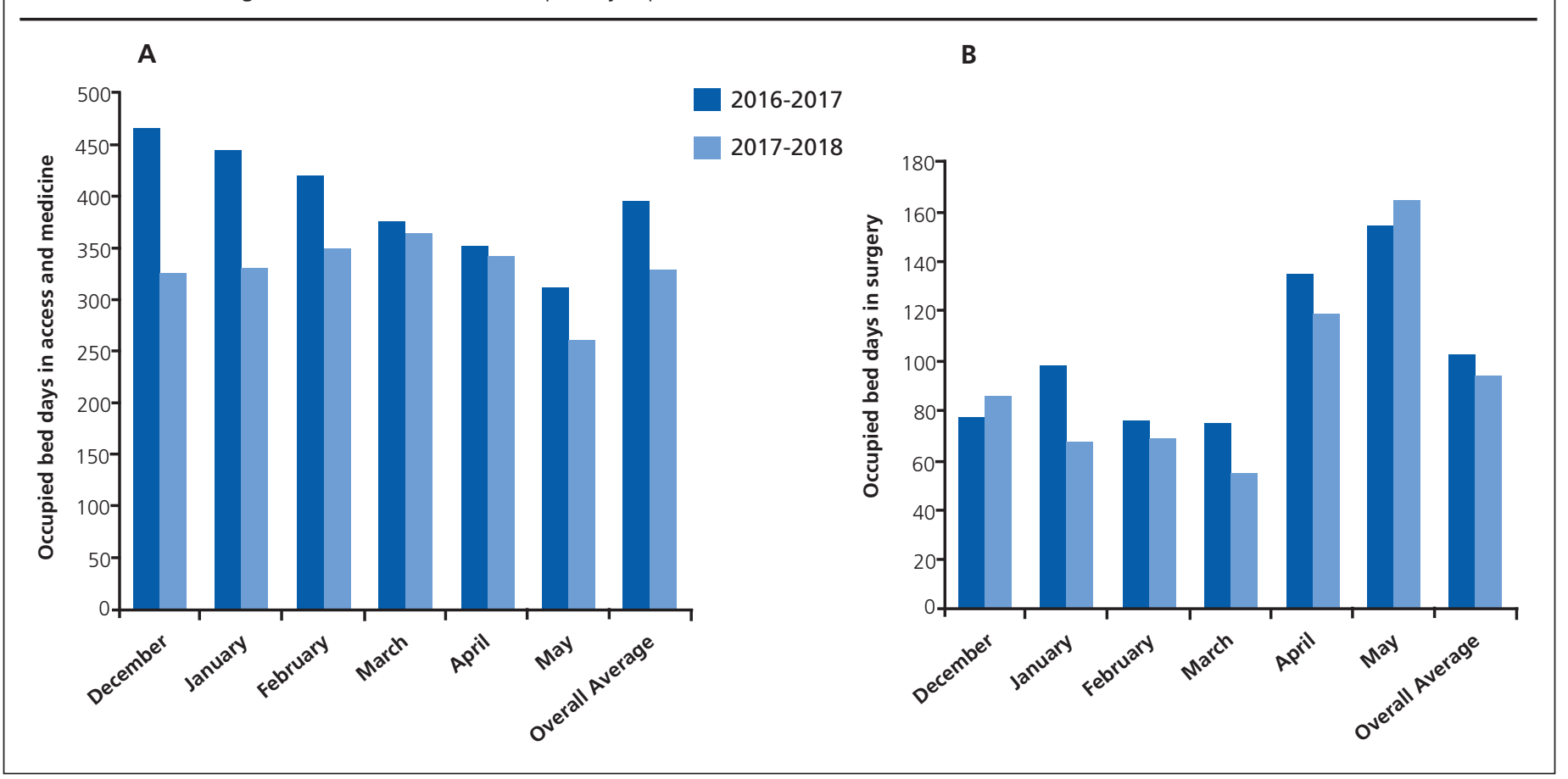

occupied bed days prior to the intervention and 8,560 occupied bed days since the intervention. In the Surgical directorate there was a total of 2,687 occupied bed days before the intervention and 2,240 occupied bed days after it. Weekly discharges increased across all directorates with a significant reduction in stranded patients (those admitted for $>7$ days). Figure 1 shows a significant reduction in occupied bed days and the considerable number of bed days that were saved in each matched month. This represents a total difference of 2,168 'saved' bed days over 6 months. The NHS Institute estimates the cost of an inpatient bed day at $f 225,{ }^{1}$ so this represents a saving of $£ 487,800$.

\section{Auditing of inpatient harms}

There was a significant reduction in Datix incident reporting and serious untoward incidents following the introduction of the inpatient diabetes service (Table 2). NaDIA-Harms data were not collected prior to the intervention but are now being audited to drive changes in practice such as the introduction of insulin recognition charts, hypoglycaemia education resources and risk assessment on prescription charts.

\section{Healthcare professional satisfaction}

An unvalidated questionnaire with measured binary questions and additional qualitative responses was undertaken 6 months after introduction of the new service. Seventy-nine members of the nursing and junior doctor staff across different specialties were asked if they felt the daily support of the diabetes team was helpful and the majority (90\%) did. Similarly, 68\% stated they felt unable to provide appropriate care for patients with diabetes without the inpatient team and their confidence to manage this patient group had increased.

Table 2 Inpatient harms

\begin{tabular}{|c|c|c|c|c|c|c|c|c|c|c|}
\hline Harms & $\begin{array}{l}\text { June- } \\
\text { Sept } 2017\end{array}$ & $\begin{array}{l}\text { Oct } \\
2017\end{array}$ & $\begin{array}{l}\text { Nov } \\
2017\end{array}$ & $\begin{array}{l}\text { Dec } \\
2017\end{array}$ & $\begin{array}{l}\text { Jan } \\
2018\end{array}$ & $\begin{array}{l}\text { Feb } \\
2018\end{array}$ & $\begin{array}{l}\text { Mar } \\
2018\end{array}$ & $\begin{array}{l}\text { April } \\
2018\end{array}$ & $\begin{array}{l}\text { May } \\
2018\end{array}$ & $\begin{array}{l}\text { June } \\
2018\end{array}$ \\
\hline $\begin{array}{l}\text { No of reported } \\
\text { incidents by } \\
\text { Diabetes Strategic } \\
\text { Business Unit }\end{array}$ & 13 & 8 & 9 & 3 & 1 & 1 & 1 & 0 & 0 & 0 \\
\hline $\begin{array}{l}\text { Serious untoward } \\
\text { incidents }\end{array}$ & 2 & 0 & 1 & 0 & 0 & 0 & 0 & 0 & 0 & 0 \\
\hline
\end{tabular}




\section{Key messages}

- The Trust-wide inpatient diabetes service redesign has shown early evidence of reducing LOS in all specialities, increasing weekly discharges and reducing diabetesrelated harm.

- The service redesign has led to improvements in governance processes and improved multi-disciplinary working

\section{Discussion}

A problem with inpatient diabetes care was recognised and characterised; consensus- and evidence-based strategies for improvement were then identified and implemented. This is the first multidisciplinary inpatient team established in a small district general hospital which has been cost neutral. In parallel, an Acute Frailty Unit was established in the ward space (with the staff of the previous general medical ward) without a loss in medical inpatient bed numbers. In addition, the diabetes consultants have maintained their General Medicine on-call commitment and outpatient activity.

The Trust-wide inpatient diabetes service redesign has shown early evidence of reducing LOS in all specialties, increasing weekly discharges and reducing diabetes-related harm. The initial LOS data have been very promising, suggesting that, since the change in practice, inpatient LOS across all divisions (Medicine and Surgery) has improved. This is in keeping with similar models enacted in other larger hospital Trusts. ${ }^{8,9} \mathrm{~A}$ reduction in LOS has been demonstrated for patients with a primary and secondary diagnosis of diabetes, offering significant cost savings.

The challenges ahead for the Trust and the inpatient service will focus on continued improvement of foot care standards in people with diabetes and targeting particular patient groups to further reduce LOS and improve glycaemia in the context of complex feeding regimes and variable perioperative glycaemic control. There is a clear governance structure and mechanism to review and develop changes in inpatient clinical care. The diabetes team visibility has improved confidence amongst healthcare professionals to manage people with diabetes and support selfmanagement, enabling improved education opportunities.

In summary, the introduction of a dedicated inpatient diabetes service has led to Trust-wide improvements in patient care and patient flow without additional cost to the Trust.

\section{Conflict of interest: None Funding: None}

\section{References}

1. Kerr M. Inpatient care for people with diabetes: the economic case for change. https://www. diabetes.org.uk/resources-s3/2017-10/Inpatient\% 20Care\%20for\%20People\%20with\%20Diabetes\%20\%20The\%20Economic\%20Nov\%202011_1.pdf

2. Health and Social Care Information Centre. National Diabetes Inpatient Audit (NaDIA) - 2017. https://digital.nhs.uk/catalogue/PUB30248 2017

3. Joint British Diabetes Societies for Inpatient Care (JBDS-IP). The Hospital Management of Hypoglycaemia in Adults with Diabetes Mellitus, 3rd edition. Revised February 2018. http://www.diabetologists-abcd.org.uk/ JBDS/JBDS_HypoGuideline_FINAL_280218.pdf (accessed March 2019)

4. National Diabetes Information Service. Diabetes Inpatient Mortality Profile Report 2010-2012. http://discovery.nationalarchives.gov.uk/details/r/ C16304248

5. Diabetes UK. Admissions avoidance and diabetes: guidance for clinical commissioning groups and clinical teams. Joint British Diabetes Societies for Inpatient Care (JBDS-IP). December 2013 http://www. diabetes.org.uk/ professionals/position-statements-reports/specialist-care-for-children-andadults-and-complications/admissions-avoidance-and-diabetes (accessed March 2019).

6. Diabetes UK. Making hospitals safe for people with diabetes. 2018. https://www.diabetes.org.uk/professionals/resources/improving-inpatientcare-programme/report-hospitals-safe

7. NHS Digital. National Diabetes In-patient Audit - Harms. https://digital.nhs.uk/data-and-information/clinical-audits-andregistries/national-diabetes-in-patient-audit-nadia-harms (accessed March 2019).

8. Bansal V, Mottalib A, Pawar TK, et al. Inpatient diabetes management by specialized diabetes team versus primary service team in non-critical care units: impact on 30-day readmission rate and hospital cost. BMJ Open Diabetes Res Care 2018;6(1):e000460. 10.1136/bmjdrc-2017-000460

9. Flanagan D, Moore E, Baker S, Wright D, Lynch P. Diabetes care in hospital--the impact of a dedicated inpatient care team. Diabet Med 2008;25(2):147-51. 10.1111/j.1464-5491.2007.02326.x 